\title{
MUSHROOM DIVERSITY OF AMRITE COMMUNITY FOREST, KAPILVASTU, NEPAL
}

\author{
Dhruv Prasad ${ }^{1}$ and Bhojraj Pokhrel ${ }^{2}$ \\ ${ }^{1}$ Skylark English Boarding School, Kapilbastu \\ ${ }^{2}$ Parijat Higher Sec. School, Kapilbastu
}

\begin{abstract}
A total of 38 wild mushroom specimens were collected for the period of 3 months (from June to September, 2016) from Amrite community forest of Bhalwad, Banganga municipality. Out of 38 species collected, 34 species were identified up to generic level belonging to 16 families. The most dominant family was Coprinaceae belonging to 5 species followed by Amanitaceae consisting of 4 species. $80 \%$ of the Tharu people were found to use mushroom as food, $12.5 \%$ as medicine and $7.5 \%$ of Tharu people don't have any idea of food and medicinal value. On the other hand, $57.5 \%$ of the non-Tharu community in the study area use mushroom as food, $7.5 \%$ use as medicine and $35 \%$ of non-Tharu community do not use mushroom as food and medicine.
\end{abstract}

Key words: community forest, mushrooms, nutrients, specimens, Tharu community,.

\section{INTRODUCTION}

Mushrooms are seasonal fungi which occupy diverse niche in nature in forest ecosystem. They predominantly occur during the rainy season. They are macromycetes forming macroscopic fruiting bodies such as Agaricus, Boletus, Jelly fungi, coral fungi, stinkhorns, bracket fungi, puffballs and bird's nest fungi. They are fleshy, sub-fleshy, or sometimes leathery and woody and bear their fertile surface either on lamellae or lining the tubes, opening out by means of pores. Mushrooms can be classified into three categories by their trophic pattern; saprophytes, parasites or mycorrhizae. The most commonly grown mushrooms are saprophytes, decomposers in an ecosystem growing on organic matters like wood, leaves and straw in nature. These varied elements have enriched Nepal with economically important mycoflora (Adhikari, 1988). Mushrooms may be edible, inedible, medicinal and poisonous. Many kinds of macro-fungi are not edible, but also possess tonic and medicinal properties (Chang and
Miles, 2004). Till now 608 genera and 2025 species have been reported under 60 order and 80 families. To date about 1021 species $(874$ species-Basidiomycotina and 147 speciesAscomycotina) of mushrooms are recorded from Nepal (Adhikari, 2012). Out of these, 228 species have food value (Christensen et al. 2008), while 73 species are medicinal and 65 species poisonous (Adhikari 2009). Wild edible mushrooms (WEMs) are important commodity to rural and tribal livelihood (Christensen et al., 2008). Mushrooms are low in calories, high in fiber, and contain many important vitamins and minerals. Some also have medicinal properties such as complex carbohydrates that strengthen the immune system Mushrooms have been recognized as delicious food of good quality protein. These are rich in vitamins particularly in vitamin $\mathrm{C}$ and vitamin B-complex and minerals. These are the food of low calorie with little fat, where sugar content is very less, starch and cholesterol are absent and ergosterol is present. 
Major mineral constituents in mushrooms are K, $\mathrm{P}, \mathrm{Na}, \mathrm{Ca}, \mathrm{Mg}$ and elements like $\mathrm{Cu}, \mathrm{Zn}, \mathrm{Fe}, \mathrm{Mo}$, $\mathrm{Cd}$ form minor constituents (Bano et al.,1982, Li and Chang,1982). K, P, Na and Mg constitute about 56 to $70 \%$ of total ash content of the mushrooms (Li and Chang, 1982).

\section{MATERIALS AND METHODS}

\section{Study Area}

The study was conducted in Amrite community forest and its vicinity that lies in Kapilbastu district in the western development region, south of the Chure hills of Argakhanchi district. Amrite community forest lies 2741' $\mathrm{N}$ and $83^{\circ} 6^{\prime} 38^{\prime \prime} \mathrm{E}$ to $27^{\circ} 44^{\prime} 46.5^{\prime \prime} \mathrm{N}$ and $83^{\circ} 05^{\prime} 48^{\prime \prime} \mathrm{E}$. It lies between the elevations of 106 to $140 \mathrm{~m}$ from mean sea level in the Kapilbastu district. Amrite community forest was established in 2066 B.S. which is conserved by more than 1200 people that includes 261 residence residing towards its southern vicinity. It is surrounded by Amrite Khola towards East, Dhire Khola towards west and south and way to Amrite cattle pasture land towards North. The forest is tropical forest which consists of Shorea robusta, Adina cordifolia, Acacia catechu, Bombax ceiba, Aegle marmelos, Anthocephalus chinensis, Terminalia alata, Terminalia chebula, Terminalia bellarica, Acorus calamus, Syzygium cuminis, Cassia fistula, etc.

\section{Data collection}

The field work was conducted five times (June to September 2016) in the "Amrite Community Forest" of the Banganga Municipality, Kapilbastu. While conducting field trips local people were also accompanied. Indigenous knowledge survey was conducted. The Participatory Rural Appraisal (PRA) technique was adopted with the local people aimed at getting information largely on nutritional aspects. Data were obtained using combined semi-structured questionnaire, participatory discussions and field observations. The mushrooms were photographed in their natural habitat before they were collected. The basidiocarps were picked up by digging them out carefully with the help of a sharp knife. Specific collection numbers were given for each species. The mushrooms were preserved by dry preserve and liquid preserve by using 95\% alcohol. The specimens were studied at Central Department of Botany, TU Kirtipur. The specimens that were collected and preserved were identified with the help of relevant literatures (Pacioni, 1985; Singer, 1986; Adhikari, 2000; Pandey, 2004; Jha et al, 2011; Jha and Tripathi, 2012; Aryal et al., 2012; Aryal and Budhathoki, 2013a, b, c, d; 2014).

\section{RESULTS AND DISCUSSION}

A total of 38 wild mushroom species were collected. Out of these 34 species were identified up to generic level belonging to 16 families. The most dominant family was Coprinaceae belonging to five species followed by Amanitaceae consisting of four species. Russulaceae, Polyporaceae, Tricholomataceae and Sclerodermataceae were represented by three species each while Agaricaceae, Boletaceae, Cantheralleceae were represented by two species each. Family Auriculariaceae, Dacrymycetaceae, Hydnangiaceae, Pannariaceae, Phallaceae, and Xylariaceae were represented by 1 species each where as four species were unidentified (Table 1).

On the basis of the information collected from 40 respondent of Tharu and 40 respondent of Non-Tharu community, $80 \%$ of the Tharu people were found to use mushroom as food, $12.5 \%$ as medicine and $7.5 \%$ of Tharu people don't have any idea of food and medicinal value. On the other hand, $57.5 \%$ of the non-Tharu community in the study area use mushroom as food, $7.5 \%$ use as medicine and $35 \%$ of non- 
Tharu community do not use mushroom as food and medicine. Thus, the above data reveals that $68.75 \%$ of the people use mushroom as food, $10 \%$ use it as medicine and $21.25 \%$ of people have no idea about the use and importance of mushroom.

According to perception of local people, it was reported that the mushrooms with following features are edible or poisonous:

- Mushrooms having annulus nearby the cap are poisonous.

- Mushroom species with curved pileus with annulus are also poisonous.

- Mushrooms found on fodder plants are generally edible.

- Mushrooms that have warts on their pileus and bad odour are poisonous.
- Mushrooms that glow at night are poisonous.

- Mushrooms that are peeled off easily are edible.

- Mushrooms that are eaten by insects and rodents are generally edible.

The edible species Auricuaria auricula reported from the present study has been already reported from Manichur (Adhikari, 1976). Pycnoporus cinnabarinus commonly known as kanya chyau has been reported from the study area but has been already reported from Ghorepani (Bhandary, 1985). The species Russula delica found on the study area has been reported from Royal Botanical Garden, Godavary (1515m) (Adhikari and Durrieu, 1996). The species Coprinus comatus reported from the study area has been reported from Chima goan.

Table 1: List of mushrooms collected from the Amrite community forest, Banganga-16, Kapilvastu.

\begin{tabular}{|c|c|c|c|c|c|c|c|}
\hline $\begin{array}{c}\text { Host/ } \\
\text { substrate }\end{array}$ & Lat./Long. & $\begin{array}{l}\text { Alt. } \\
\text { (m) }\end{array}$ & Scientific name & Local name & Order & Family & Application \\
\hline soil & $27^{\circ} 41^{\prime} 01.5^{\prime \prime} / 83^{\circ} 06^{\prime} 38^{\prime \prime}$ & 114 & Macrolapiota procera (Scop.) Singer & Gobre chyau & Agaricales & Agaricaceae & Non-edible \\
\hline soil & $27^{\circ} 41^{\prime} 11.5^{\prime \prime} / 83^{\circ} 05^{\prime} 36^{\prime \prime}$ & 112 & Tricholar sp. & & Agaricales & Tricholomataceae & Medicinal \\
\hline soil & $27^{\circ} 41^{\prime} 12.5^{\prime \prime} / 83^{\circ} 06^{\prime} 28^{\prime \prime}$ & 122 & Russula spp. & Raktey & Russulales & Russulaceae & Edible \\
\hline soil & $27^{\circ} 41^{\prime} 21^{\prime \prime} / 83^{\circ} 06^{\prime} 48^{\prime \prime}$ & 121 & Russula emetica (Schaeff.) Pers. & Kali Chyau & Russulales & Russulaceae & Edible \\
\hline soil & $27^{\circ} 42^{\prime} 22^{\prime \prime} / 83^{\circ} 08^{\prime} 38^{\prime \prime}$ & 136 & $\begin{array}{l}\text { Pycnoporus cinnabarinus ( } \underline{\text { Jacq. }} \underline{\text { P. }} \\
\underline{\text { Karst. }}\end{array}$ & Kane Chyau & Polyporales & Polyporaceae & Medicinal \\
\hline soil & $27^{\circ} 41^{\prime} 25^{\prime \prime} / 83^{\circ} 06^{\prime} 14^{\prime \prime}$ & 127 & Scleroderma texense Berk. & Bhutki & Bolatales & Sclerodermataceae & Edible \\
\hline soil & $27^{\circ} 42^{\prime} 17^{\prime \prime} / 83^{\circ} 06^{\prime} 36^{\prime \prime}$ & 110 & Coprinus Commatus (․․Müll.) Pers. & Gobre & Agaricales & Coprinaceae & Unknown \\
\hline Soil & $27^{\circ} 41^{\prime} 03^{\prime \prime} / 83^{\circ} 05^{\prime} 44^{\prime \prime}$ & 122 & $\begin{array}{ll}\text { Amanita chepangiana } \\
\text { Tulloss and Bhandary }\end{array}$ & Salle Chyau & Agaricales & Amanitaceae & Edible \\
\hline Soil & $27^{\circ} 41^{\prime} 50^{\prime \prime} / 83^{\circ} 05^{\prime} 22^{\prime \prime}$ & 112 & Buwaldo boletus spp. & $\begin{array}{l}\text { D h a b r e } \\
\text { chyau }\end{array}$ & Bolatales & Boletaceae & Poisonous \\
\hline soil & $27^{\circ} 43^{\prime} 19^{\prime \prime} / 83^{\circ} 05^{\prime} 07^{\prime \prime}$ & 112 & Scleroderma cepa Pers. & Bhutki & Bolatales & Sclerodermataceae & Edible \\
\hline soil & $27^{\circ} 44^{\prime} 01^{\prime \prime} / 83^{\circ} 05^{\prime} 20^{\prime \prime}$ & 114 & Amanita sp. & $\begin{array}{l}\text { B e s e r a } \\
\text { Cheyu }\end{array}$ & Agaricales & Amanitaceae & Edible \\
\hline Soil & $27^{\circ} 42^{\prime} 05^{\prime \prime} / 83^{\circ} 05^{\prime} 20^{\prime \prime}$ & 118 & Russula spp. & $\begin{array}{l}\text { R a t t e u o } \\
\text { Chyau }\end{array}$ & Russulales & Russulaceae & Edible \\
\hline Soil & $27^{\circ} 42^{\prime} 44^{\prime \prime} / 83^{\circ} 05^{\prime} 16^{\prime \prime}$ & 140 & Boletus spp. & $\begin{array}{l}\text { B h u d e } \\
\text { Chyau }\end{array}$ & Bolatales & Boletaceae & Medicinal \\
\hline wood & $27^{\circ} 41^{\prime} 01.5^{\prime \prime} / 83^{\circ} 06^{\prime} 38^{\prime \prime}$ & 114 & Sparissis crispa (Wolfen) Fr. & Cauli Chyau & Polyporale & Sparassidaceae & \\
\hline wood & $27^{\circ} 42^{\prime} 15^{\prime \prime} / 83^{\circ} 0631^{\prime \prime}$ & 123 & Guepinia spathularia (Schwein.) Fr. & Putali Chyau & Tramellales & Dacrymycetacea & \\
\hline wood & $27^{\circ} 41^{\prime} 11.5^{\prime \prime} / 83^{\circ} 05^{\prime} 36^{\prime \prime}$ & 112 & Nebularia sp. & & Peltigerales & Pannariaceae & \\
\hline
\end{tabular}




\begin{tabular}{|c|c|c|c|c|c|c|c|}
\hline dung & $27^{\circ} 42^{\prime} 22^{\prime \prime} / 83^{\circ} 05^{\prime} 06^{\prime \prime}$ & 132 & Coprinus plicalitis Fr. ex Curtis & Gobre Chyau & Agaricales & Coprinaceae & Inedible \\
\hline soil & $27^{\circ} 42^{\prime} 44^{\prime \prime} / 83^{\circ} 05^{\prime} 16$ & 140 & $\begin{array}{l}\text { Termitomyces aurantiacus (R Heim) } \\
\text { R. Heim }\end{array}$ & $\begin{array}{l}\text { D h a m i r e } \\
\text { Chyau }\end{array}$ & Agaricales & Tricholomataceae & \\
\hline soil & $27^{\circ} 42^{\prime} 05^{\prime \prime} / 83^{\circ} 05^{\prime} 20^{\prime \prime}$ & 118 & Lacaria laccata ( $\underline{\text { Scop. }}$ ) Cooke & $\begin{array}{l}\text { D u d h e } \\
\text { Chyau }\end{array}$ & Agaricales & Hydnangiaceae & \\
\hline $\begin{array}{l}\mathrm{t} r \mathrm{e} \mathrm{e} \\
\text { trunk }\end{array}$ & $27^{\circ} 42^{\prime} 05^{\prime \prime} / 83^{\circ} 05^{\prime} 20^{\prime \prime}$ & 118 & Polyprous durus & Kathe chyeu & Polyporales & Polyporaceae & Edible \\
\hline $\begin{array}{l}\mathrm{t} r \mathrm{e} \mathrm{e} \\
\text { trunk }\end{array}$ & $27^{\circ} 42^{\prime} 44^{\prime \prime} / 83^{\circ} 05^{\prime} 16$ & 140 & $\begin{array}{l}\text { Auricularia auricula-jude (Bull.) J. } \\
\text { Schröt. }\end{array}$ & Todke & Agaricales & Auriculariaceae & Medicinal \\
\hline soil & $27^{\circ} 41^{\prime} 11.5^{\prime \prime} / 83^{\circ} 05^{\prime} 36^{\prime \prime}$ & 112 & unidentified & & & & \\
\hline Wood & $27^{\circ} 42^{\prime} 22^{\prime \prime} / 83^{\circ} 05^{\prime} 06^{\prime \prime}$ & 132 & Microporous xanthpus & Kathe chyau & Polyporales & Polyporaceae & Medicinal \\
\hline wood & $27^{\circ} 42^{\prime} 05^{\prime \prime} / 83^{\circ} 05^{\prime} 20^{\prime \prime}$ & 118 & Daldenia sp. & Dalley & Polyporales & Xylariaceae & \\
\hline Soil & $27^{\circ} 41^{\prime} 01.5^{\prime \prime} / 83^{\circ} 06^{\prime} 38^{\prime \prime}$ & 114 & $\begin{array}{l}\text { Amanita } \\
\text { (Berk. and } \underline{\text { Broome }} \text { ) } \underline{\text { Sacc. }}\end{array}$ & Salle chyau & Agaricales & Amanitaceae & Edible \\
\hline Wood & $27^{\circ} 44^{\prime} 02^{\prime \prime} / 83^{\circ} 0633^{\prime \prime}$ & 129 & Cantharellus sp. & $\begin{array}{l}\mathrm{C} \mathrm{h} \mathrm{a} \mathrm{m} \mathrm{re} \\
\text { Chyaue }\end{array}$ & Cantharellales & Cantharellacae & Edible \\
\hline Soil & $27^{\circ} 42^{\prime} 15^{\prime \prime} / 83^{\circ} 06^{\prime} 31^{\prime \prime}$ & 123 & Amanita sp. & Salle & Agaricales & Amanitaceae & Poisonous \\
\hline Rock & $27^{\circ} 44^{\prime} 02^{\prime \prime} / 83^{\circ} 0633^{\prime \prime}$ & 129 & Unidentified & & & & \\
\hline Soil & $27^{\circ} 42^{\prime} 44^{\prime \prime} / 83^{\circ} 05^{\prime} 16$ & 140 & Marasmius perforans (Hoffm.) Fr. & $\begin{array}{l}\text { B u } 1 \text { a k i } \\
\text { Chyau }\end{array}$ & Agaricales & Tricholomataceae & \\
\hline soil & $27^{\circ} 44^{\prime} 02^{\prime \prime} / 83^{\circ} 06^{\prime} 33^{\prime \prime}$ & 129 & Unidentified & Kukurmuta & & & Inedible \\
\hline $\begin{array}{l}\text { animal } \\
\text { dung }\end{array}$ & $27^{\circ} 42^{\prime} 22^{\prime \prime} / 83^{\circ} 05^{\prime} 06^{\prime \prime}$ & 138 & Coprinus atramentarius (Bull.) $\mathrm{Re}$ & Gobre Chyau & Agaricales & Coprinaceae & \\
\hline $\begin{array}{l}\text { animal } \\
\text { dung }\end{array}$ & $27^{\circ} 41^{\prime} 11.5^{\prime \prime} / 83^{\circ} 05^{\prime} 36^{\prime \prime}$ & 112 & Agaricus sp. & Gobre chyau & Agaricales & Agaricaceae & Edible \\
\hline Soil & $27^{\circ} 42^{\prime} 22^{\prime \prime} / 83^{\circ} 05^{\prime} 06^{\prime \prime}$ & 132 & Scleroderma bovista Fr. & Dalle chyau & Boletales & Sclerodermataceae & Medicinal \\
\hline Soil & $27^{\circ} 41^{\prime} 01.5^{\prime \prime} / 83^{\circ} 0638^{\prime \prime}$ & 114 & Cantharellus cibarius Fr. & Besare chyau & Cantharellales & Cantherallaceae & Edible \\
\hline Soil & $27^{\circ} 44^{\prime} 02^{\prime \prime} / 83^{\circ} 0633^{\prime \prime}$ & 129 & Dictyophora indusiata (Vent.) Desv. & Jali Chyau & Phallales & Phallaceae & Poisonous \\
\hline
\end{tabular}

C.date $=$ Collection date, Lat. $=$ Latitude, Long. $=$ Longitude, Alt. $=$ Altitude 


\section{Photo plates}

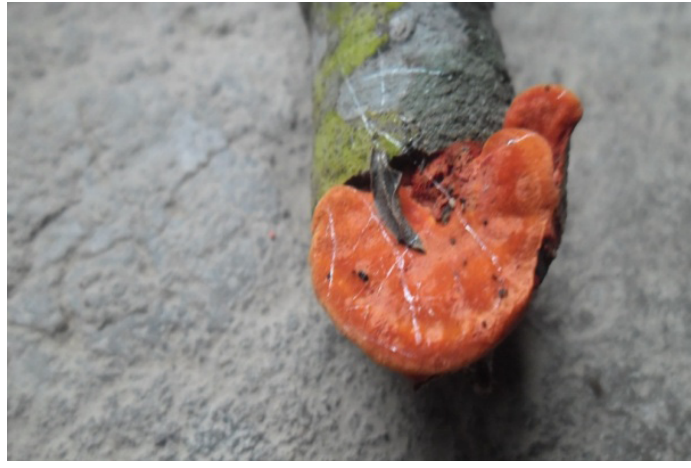

Pycnoporous cinnabarinus (Jacq.:Fr)Karst.

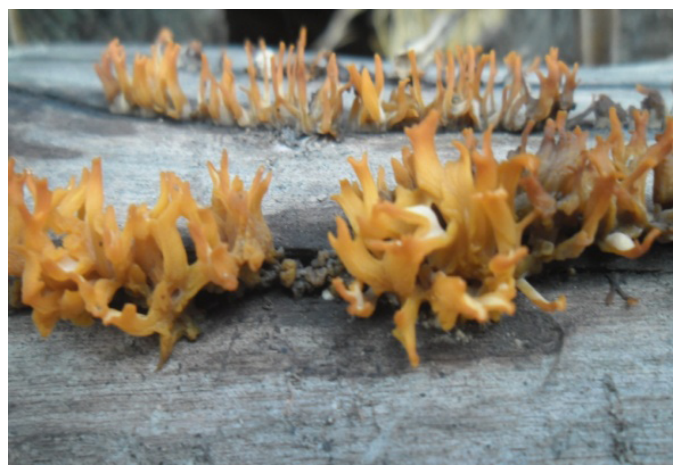

Guepinia spathularia (Schwein.) Fr.

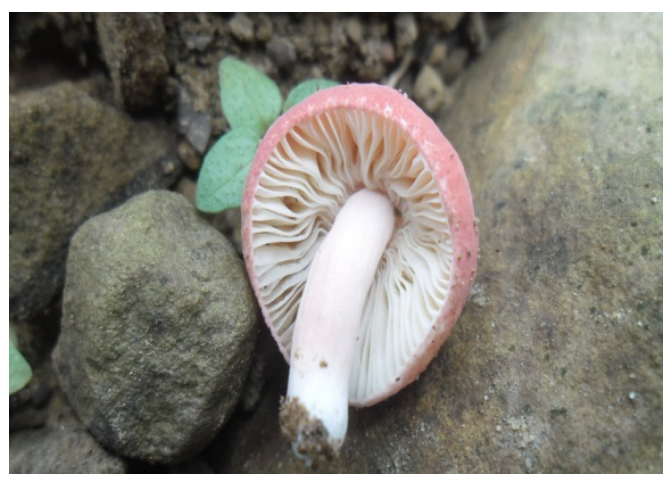

Russula emitica (Sch.:Fr)Pers.

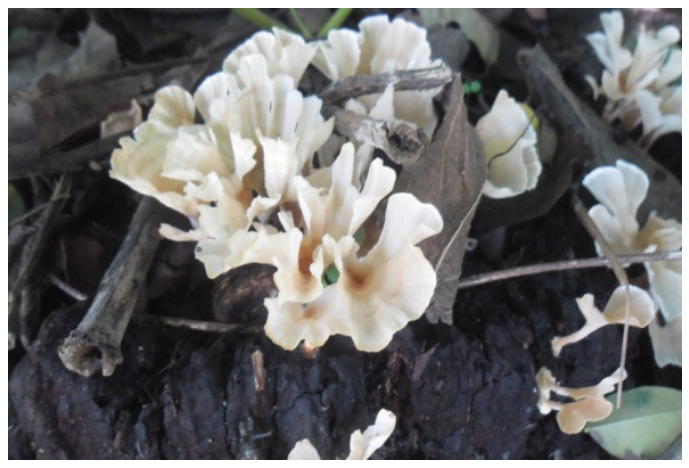

Sparassis crispa (Wolfen) Fr.

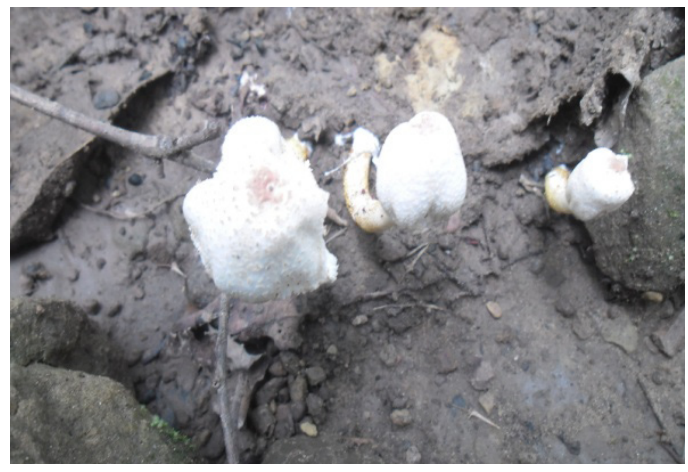

Macrolapiota (Scop.) Singer

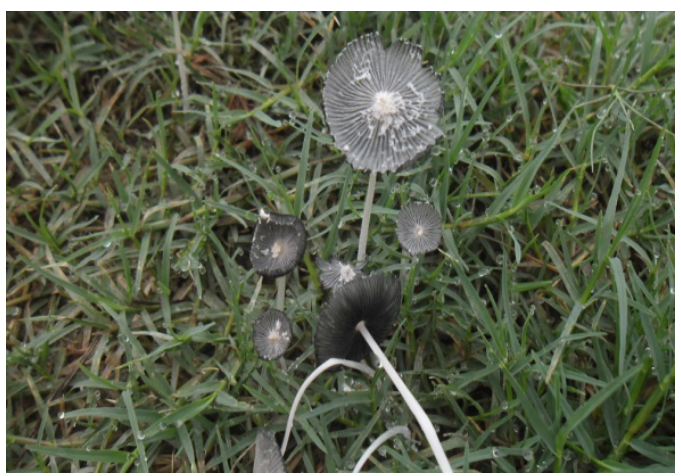

Coprinus plicalitis Fr. ex Curtis 


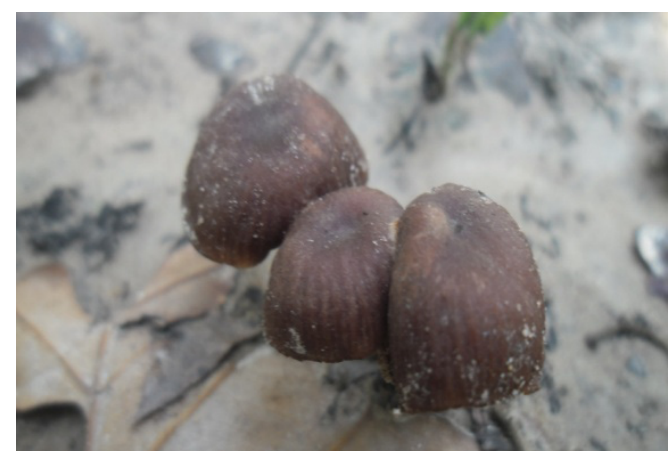

Lacaria laccata (Scop.) Cooke

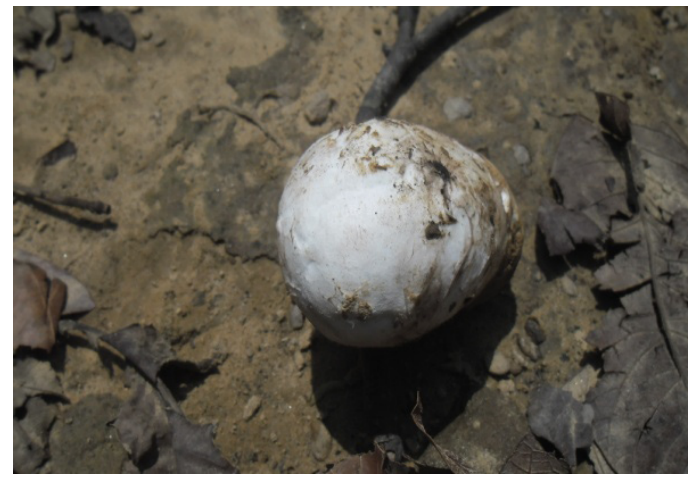

Amanita chapengiana Tulloss and Bhandary

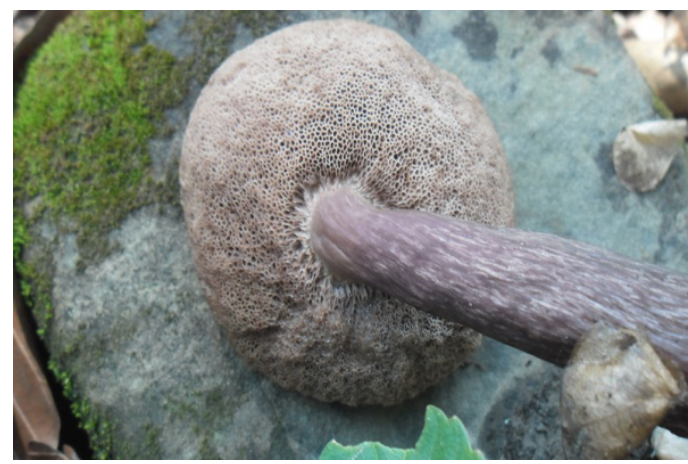

Buwaldo boletus spp.

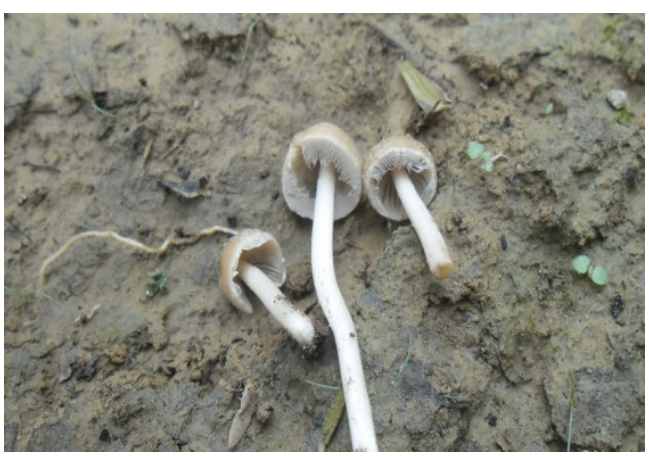

Coprinus disseminates (Pers.) J.E.Lange

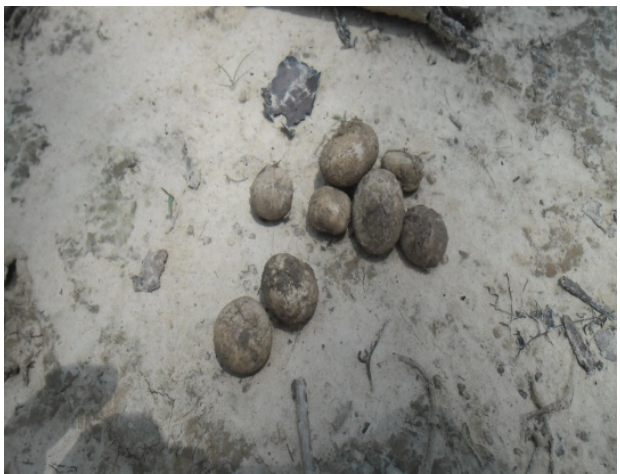

Scleroderma taxiens Berk.

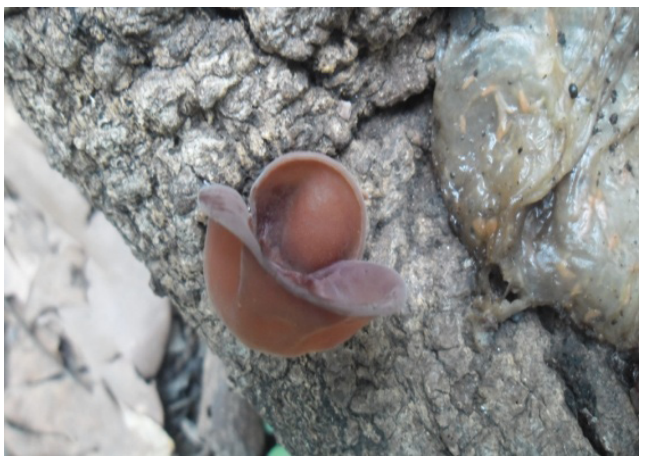

Auricularia auricula-jude (Bull.) J.Schröt. 


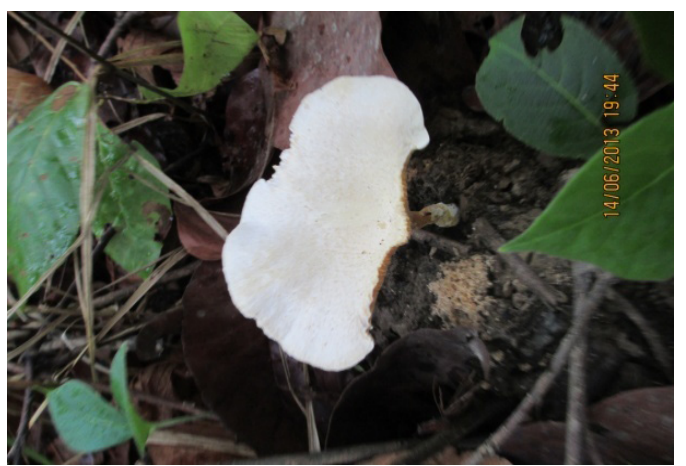

Polyporus durus (Timm.)Kreisel.

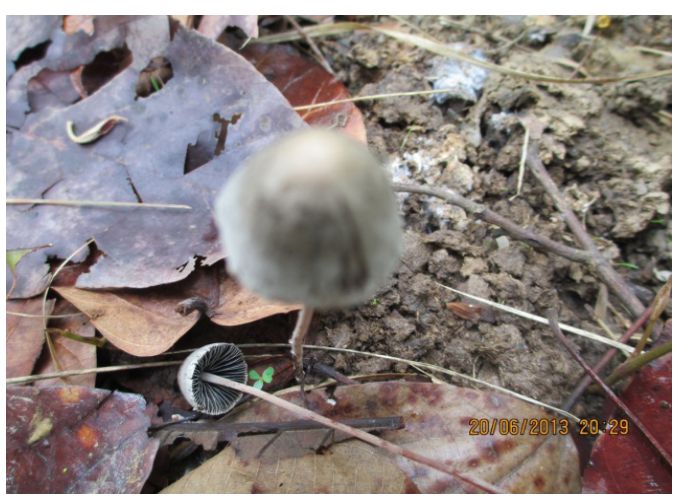

Coprinus comatus (Mull.:Fr)Pers.

\section{ACKNOWLEDGMENTS}

The Authors are thankful to Dr. Hari Prasad Aryal for his kind help in the identification of mushrooms. Also, the authors are thankful to Prof. Dr. Chandra Bahadur Thapa and Dr. Anant Gopal Singh for publishing this article.

\section{REFERENCES}

Adhikari, M.K. (1976). Chyau: ek Charcha. Gorkhapatra (Kath.) 76: 6 (in Nepali (2033.8.10).

Adhikari, M. K. (1988). Polypores (wood rotting fungi) of Nepal. Banko Janakari, 2(1): 9-20.

Adhikari, M.K. and G. Durrieu, (1996). Ethnomycologie népalaise. Bull. Soc. Mycol. France, 112 : 31-41.

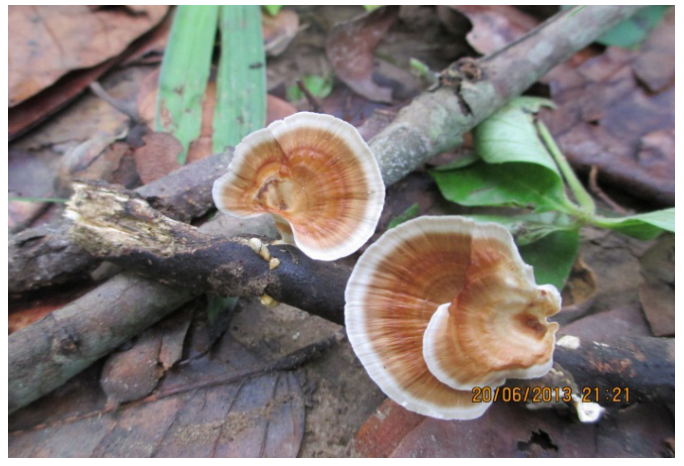

Microporus xanthopus (Fr.) Kuntze

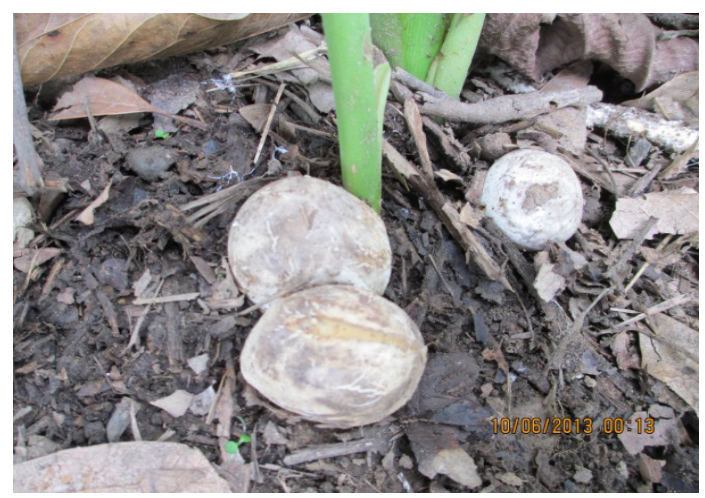

Dictophora indusiata (Vent.) Desv.

Adhikari, M. K. (2000). Mushrooms of Nepal. Kathmandu, Nepal: P.U. Printers.

Adhikari, M. K. (2009). Researches on the Nepalese Mycoflora: revised account on the history of mycological explorations. Kathmandu, Nepal: K.S. Adhikari.

Adhikari, M. K. (2012). Researches on the Nepalese Mycoflora - 2: Checklist of macrofungi (mushrooms). Kathmandu, Nepal: K.S. Adhikari.

Aryal, H. P., U. Budhathoki, and M. K. Adhikari (2012). Mucodiversity of Pipaldanda Community Forest, Western Terai Region of Nepal. (19958579). Kathmandu, Nepal: Government of Nepal, Ministry of Soil and Forest conservation.

Aryal, H.P. and U. Budhathoki, (2013) a. Buchwaldobolems lignicola (Basidiomcetes) an Inedible Wild Mushroom New to Nepal. Our 
Nature, 11(1): 31-35.

Aryal, H. P. and U. Budhathoki,2013b. The Genus Amanita (Pers.). In Lumbini Zone Nepal. Scientific World, 11(1): 113-120.

Aryal H.P. and U. Budhathoki, 2013c.Mycodivesity of shankarnagar community forest, Rupandehi District. Nep. Jour. Sc. Tech. 2013, 14 (1).

Aryal, H.P. and U. Budhathoki, $2013 \mathrm{~d}$. Ethnomycological studies on some macro-fungi in Rupandehi District, Nepal. Banko Janakari, 2013. 23 (1):51-56.

Aryal , H.P. and U. Budhathoki, (2014). Some wild mushroom of Rupandehi District, west Nepal. BIBECHANA. A Multidisciplinary Journal of science, technology and mathematics. ISSN 2091-0762.10 (2014) 34-43:BMHSS. P. 34.

Bano Z, and S. Rajarathanam (1982). Pleurotus mushrooms as a nutritious food. In: Tropical mushrooms -Biological nature and cultivation methods. Chang ST, Quimio TH (eds). The Chinese University press, Hongkong. pp. 363382.

Bhandary, H. R. (1985). Nepalka Chayuharu. Bull. Nast. Hist. Mus., 1, 17-19.

Bisaria R., M. Madan, V.S. Bisaria (1987). Biological efficiency and nutritive value of $P$. sajor-caju cultivated on different agrowastes. Biological Wastes. 19:239-255.

Chang, S.T. and P.G. Miles. (2004). Mushrooms: Cultivation, Nutritional Value, Medicinal Effect, and Environmental Impact (Second Edition). CRC Press. Boca Raton, 451p.

Christensen, M., S. Bhhatarai, S. Devakota and H.O. Larsen (2008). Collection and use of wild edible fungi in Nepal. Economic Botany, 62(1):12-23.

Giri, A., and P. Rana (2008). Ethnomycological Knowledge and Nutritional Analysis of Some Wild Edible Mushrooms of Sagarmatha National Park (SNP), Nepal. $J$. Nat. His. Mus., 23:65-77.
Jha, S. K., N. Kumar and N. N. Tripathi (2011). Survey Of Ethnomedicinal Macrofungi of Nagarjun and Phulchowki Areas of Kathmandu Valley, Nepal. International Journal of Pharmaceutical Sciences Review and Research, 11(1), 147-151.

Jha, S. K., and N. N. Tripathi (2012). Diversity of Macrofungi in Shivapuri National Park of Kathmandu Valley, Nepal. Biological Forum-An International Journal, 4(1), 2734.

Li, G.S.F. and S.T. Chang (1982). Nutritive of Volveriella volvaceae. In: Tropical mushrooms-Biological nature and cultivation methods (Chang ST, Quimio TH (eds.)) Chinese University press Hong Kong, Pp. 199-219.

Pacioni, G. (1985). The Mcdonald's encyclopedia of mushrooms and toadstools. London: Mcdonald and Co. Ltd.

Pandey, N. (2004). Chemical analysis of mushrooms of Kathmandu valley. A report submitted to University Grants Commission (UGC), Kathmandu. Pp59.

Singer, R. (1986). The Agaricales in Modern Taxonomy. $4^{\text {th }}$ edition Bishen Singh and Mahendra Pal Singh, Dehradun, India.

(Received 12 May 2017, revised accepted 14 July 2017) 